\title{
Development of a Label for Green Software Products: A person-oriented view
}

\author{
Eva Kern \\ Leuphana University Lueneburg, Lueneburg, Germany \\ Environmental Campus Birkenfeld, Birkenfeld, Germany \\ mail@nachhaltige-medien.de
}

\section{INTRODUCTION}

Information and communication technologies (ICT) consume a lot of natural resources and influence the life of sociality in many different contexts. Thus, academia came up with research projects addressing sustainability issues of ICT. Generally, these comprise social, economic and environmental aspects. Some approaches add an individual and a technical dimension. The last two dimensions refer to issues of human capital (individual sustainability) and to durability of technical systems (technical sustainability). [1] Overall, most of the activities seem to deal with questions regarding environment so far.

Relative to research regarding "Green IT" mainly focusing on hardware issues the consideration of the software side is a new research field. It seems as if the relevance of integrating the software side into improvements of the ICT energy consumption and sociality development has arrived in the research community. Here, different suggestions on how to define "green" or "sustainable software" can be found (e.g. [2, 3]). Above, there are, among others, approaches for sustainability issues in requirement engineering $[4,5]$, corresponding procedure models $[3,6]$ and measurement methods $[7,8]$.

In order to reach a transformation in ICT towards a more sustainable way of developing, distributing, using, deactivating and disposing of software, it is necessary to include everyone who is related to these life cycle phases of a software product [9]. Next to the research activities, this contains the practical and personal side. The question arises how to inform the nonacademic stakeholders about sustainability issues of software. One idea is to develop a label for these kind of software and thus create more transparency, hopefully interest and greater awareness regarding these aspects. In the long term this might lead to an attitude change in software aspects.

\section{CONTENT OF THE POSTER}

The presented poster lays an emphasis on the environmental dimension of sustainability informatics. This is caused by the idea of focusing on one aspect in the first run to be able to concentrate on this dimension instead of overloading the information being presented by a more comprehensive label for software products. Hence, the object of interest is an eco-label for software here.
The following questions are addressed by the poster:

- Who are the stakeholders in the development process of a label for green software products?

- What is the role of the end user within the label development process?

- How to integrate the end user into the development process?

Possible stakeholders in the context of sustainability, green software engineering and Green IT are discussed and presented by Penzenstadler et al. [1], Naumann et al. [9] and Herzog et al. [10]. These results have been taken as starting point to extract potential stakeholders in the development process of a label for green software products. While every one of them plays his own role in the development process, the end users are analyzed in more detail here: they are assigned to different roles that explain why the end users presents an important part in developing an eco-label for software products. Above, first approaches how to integrate the end user into the development process of a label for green software products are suggested. They can be distinguished by the number of integrated persons (e.g. a survey can be spread to and answered by many users whereas just some persons can join a transdisciplinary research project) and involvement (e.g. the users get the chance to contribute the label process in a greater extend while participating in a project than filling out a survey).

\section{REFERENCES}

[1] Penzenstadler B, Femmer H, Richardson D (2013) Who is the advocate? stakeholders for sustainability. In: Green and Sustainable Software (GREENS), 2013 2nd International Workshop on, pp 70-77.

[2] Penzenstadler B, Raturi A, Richardson D et al. (2014) Systematic Mapping Study on Software Engineering for Sustainability (SE4S) - Protocol and Results, Irvine.

[3] Dick M, Naumann S (2010) Enhancing Software Engineering Processes towards Sustainable Software Product Design. In: Greve K, Cremers AB (eds) EnviroInfo 2010: Integration of Environmental Information in Europe: Proceedings of the 24th International Conference on Informatics for Environmental Protection, October 6 - 8, 2010, Cologne/Bonn, Germany. Shaker, Aachen, pp 706-715. 
[4] Mahaux M, Canon C (2012) Integrating the complexity of sustainability in requirements engineering. In: First international workshop on Requirements for Sustainable Systems.

[5] Penzenstadler B, Mahaux M, Salinesi C (2013) RE4SuSy: Requirements Engineering for Sustainable Systems. Journal of Systems and Software.

[6] Lami G, Fabbrini F, Fusani M (2013) A Methodology to Derive Sustainability Indicators for Software Development Projects. In: Proceedings of the 2013 International Conference on Software and System Process. ACM, New York, NY, USA, pp 70-77.

[7] Corral L, Georgiev AB, Sillitti A et al. (2013) A method for characterizing energy consumption in Android smartphones. In:
Green and Sustainable Software (GREENS), 2013 2nd International Workshop on, pp 38-45.

[8] Capra E, Francalanci C, Slaughter SA (2012) Measuring Application Software Energy Efficiency. IT Professional: 5461.

[9] Naumann S, Dick M, Kern E et al. (2011) The GREENSOFT Model: A Reference Model for Green and Sustainable Software and its Engineering. SUSCOM 1(4): 294-304. doi: 10.1016/j.suscom.2011.06.004.

[10] Herzog C, Lefévre L, Pierson J (2015) Actors for Innovation in Green IT. In: Hilty LM, Aebischer B (eds) ICT Innovations for Sustainability: Advances in Intelligent Systems and Computing. Springer, pp 49-67. 